\title{
Lateral lithiation and substitution of $N^{\prime}$-(2-methylphenyl)- $N, N$-dimethylurea
}

\author{
Keith Smith, ${ }^{* a}$ Gamal A. El-Hiti, ${ }^{b}$ Sadiq A. Al-Mansury, ${ }^{c}$ \\ Mohammed B. Alshammari, ${ }^{d}$ and Asim A. Balakit ${ }^{e}$ \\ a School of Chemistry, Cardiff University, Main Building, Park Place, \\ Cardiff, CF10 3AT, UK \\ ${ }^{b}$ Cornea Research Chair, Department of Optometry, College of Applied Medical Sciences, \\ King Saud University, P.O. Box 10219, Riyadh 11433, Saudi Arabia \\ ${ }^{c}$ Department of Chemistry, College of Veterinary Medicine, Al-Qasim Green University, \\ Babil, Iraq \\ ${ }^{d}$ Chemistry Department, College of Sciences and Humanities, Salman bin Abdulaziz University, \\ P.O. Box 83, Al-Kharij 11942, Saudi Arabia \\ e College of Pharmacy, Babylon University, Babylon, Iraq \\ E-mail: smithk13@cardiff.ac.uk,gelhiti@ksu.edu.sa
}

DOI: $\underline{\text { http://dx.doi.org/10.3998/ark.5550190.p008.800 }}$

\begin{abstract}
Lithiation of $N^{\prime}$-(2-methylphenyl)- $N, N$-dimethylurea with three molar equivalents of tert-butyllithium at -40 to $-30{ }^{\circ} \mathrm{C}$ takes place on the nitrogen and on the methyl group at position 2 of the phenyl group. The lithium intermediate thus obtained reacts with a variety of electrophiles to give the corresponding side-chain substituted derivatives in high yields.
\end{abstract}

Keywords: Lateral lithiation, $N^{\prime}$-(2-methylphenyl)- $N, N$-dimethylurea, lithium intermediate

\section{Introduction}

Lateral lithiation, followed by reactions with electrophiles, provides a convenient route for the production of substituted aromatics and heterocycles. Such lateral lithiation requires a group that stabilizes an organolithium either by coordination or by delocalizing a negative charge. ${ }^{1,2}$ Various heteroatom-based stabilizing groups, located at an ortho-position, have been used successfully for lateral lithiations. ${ }^{3-16}$

In the course of our own studies of lithiation reactions ${ }^{17}$ we have synthesized various substituted aromatics and heterocycles via efficient lateral lithiation procedures. ${ }^{18-22}$ For example, we have successfully laterally lithiated and substituted $N^{\prime}$-(2-methylbenzyl)- $N, N$ - 
dimethylurea (1) with tert-butyllithium (t-BuLi; 2.2 equiv.) at $-78{ }^{\circ} \mathrm{C}$ in tetrahydrofuran (THF) to produce the corresponding substituted derivatives 2 in high yields (Scheme 1). ${ }^{21}$

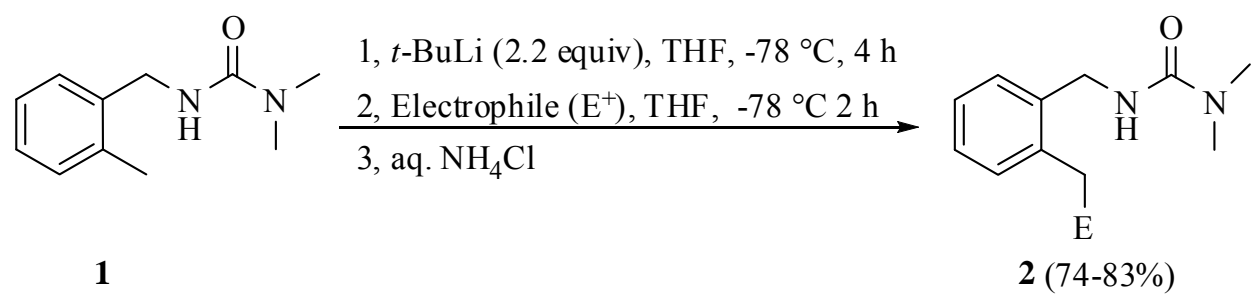

Scheme 1. Lateral lithiation and substitution of $N^{\prime}$-(2-methylbenzyl)- $N, N$-dimethylurea (1). ${ }^{21}$

Recently, we have shown that lithiation of $N^{\prime}$-(2-(2-methylphenyl)ethyl)- $N, N$-dimethylurea (3) with $n$-butyllithium ( $n$-BuLi; 3.0 equiv.) at $0{ }^{\circ} \mathrm{C}$ in THF, rather than taking place on the methyl group, takes place on the $\mathrm{CH}_{2}$ next to the 2-methylphenyl ring ( $\alpha$-lithiation), giving substituted derivatives 4 in excellent yields following in-situ reaction with electrophiles (Scheme $2) .^{22}$

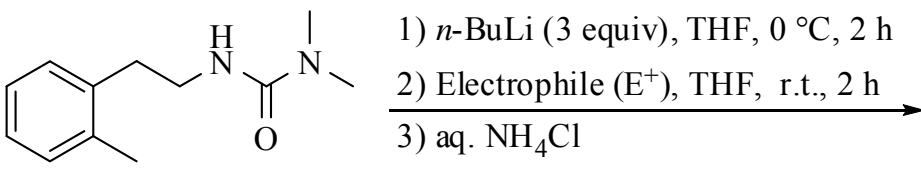

3

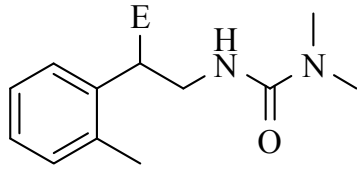

$4(78-93 \%)$

Scheme 2. Lithiation and substitution of $N^{\prime}$-(2-(2-methylphenyl)ethyl)- $N, N$-dimethylurea (3). ${ }^{22}$

There are no previous reports of lithiation and substitution of $N^{\prime}$-(2-methylphenyl)- $N, N$ dimethylurea. We now report that lithiation of this compound takes place on the methyl group at position 2 to provide substituted derivatives that might have pharmacological activities and would be difficult to prepare by other means.

\section{Results and Discussion}

$N^{\prime}$-(2-Methylphenyl)- $N, N$-dimethylurea (6) was synthesized in $99 \%$ yield after crystallization, based on a literature procedure for analogous compounds, ${ }^{21,22}$ from reaction of 2-toluidine (5) with dimethylcarbamoyl chloride (DMCC) under reflux for $2 \mathrm{~h}$ in dichloromethane (DCM) in the presence of triethylamine (TEA) (Scheme 3). The spectroscopic data for 6 were consistent with 
those reported for the product of reaction of 2-tolyl isocyanate with dimethylamine hydrochloride. $^{23}$<smiles>Cc1ccccc1N</smiles>

5

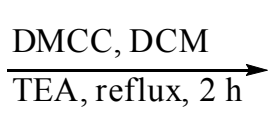

TEA, reflux, $2 \mathrm{~h}$<smiles>Cc1ccccc1NC(=O)N(C)C</smiles>

$6(99 \%)$

Scheme 3. Synthesis of $N^{\prime}$-(2-methylphenyl)- $N, N$-dimethylurea (6).

Initially the reaction of 6 with $n$ - BuLi (2.5 equiv.) was carried out in anhydrous THF at $-78{ }^{\circ} \mathrm{C}$ under a nitrogen atmosphere. Initial addition of $n$-BuLi provided a pale yellow solution, presumably because of formation of the monolithium reagent 7 (Scheme 4), until approximately one equivalent had been added, then gave a deep yellow solution as the remaining $n$-BuLi was added, presumably because of formation of a dilithium reagent. The mixture was stirred at -78 ${ }^{\circ} \mathrm{C}$ for $2 \mathrm{~h}$. Benzophenone (1.2 equiv.) was added, the mixture was stirred for another $2 \mathrm{~h}$ at -78 ${ }^{\circ} \mathrm{C}$ and the reaction was then quenched by the addition of aqueous ammonium chloride $\left(\mathrm{NH}_{4} \mathrm{Cl}\right)$ solution. The ${ }^{1} \mathrm{H}$ NMR spectrum of the product mixture showed that $N$ '-(2-(2-hydroxy-2,2diphenylethyl)phenyl)- $N, N$-dimethylurea (9) was produced, but in only $c a$. $7 \%$ yield (Table 1 ; Entry 1), along with residual 6 (ca. 90\%). This implied that the expected laterally lithiated reagent 8 was produced in-situ (Scheme 4), although in low yield. Use of $t$-BuLi as the lithiating agent under similar reaction conditions provided no product and only starting material 6 was quantitatively recovered (Table 1; Entry 2).

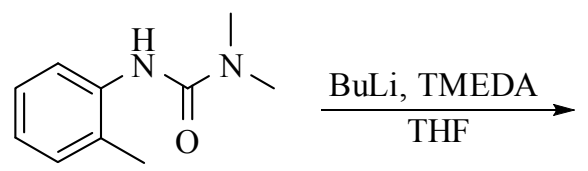

6<smiles>CN(C)C(=O)Nc1ccccc1CC(O)(c1ccccc1)c1ccccc1</smiles><smiles>Cc1ccccc1/N=C(\O)N(C)C</smiles>

7<smiles>CN(C)C(=Nc1ccccc1C[Al])O[AlH2]</smiles>

Scheme 4. Lithiation of 6 followed by reaction with benzophenone. 
Raising the temperature of lithiation to $-20{ }^{\circ} \mathrm{C}$ improved the yield of product 9 to $17 \%$ ( $n$ $\mathrm{BuLi}$ ) and 31\% (t-BuLi), respectively (Table 1; Entries 3 and 4), although there was still much residual 6. However, the NMR spectra of the product mixtures showed the presence of traces of a side-product. Raising the temperature of lithiation to $0{ }^{\circ} \mathrm{C}$ failed to provide any of the substituted product 9 (Table 1; Entries 5 and 6), but the side-product became significant, so it was purified by column chromatography (silica; EtOAc) and then identified as $\mathbf{1 0}$ (16\% yield with $n$-BuLi and $39 \%$ with $t$-BuLi). Production of $\mathbf{1 0}$ involves incorporation of two additional carbon atoms and Clayden has shown that conditions similar to those used in these reactions result in significant formation of acetaldehyde enolate by organolithium-induced decomposition of THF. ${ }^{24}$ Assuming the enolate to be the source of the additional carbon atoms, the mechanism shown in Scheme 5 is suggested for the formation of $\mathbf{1 0}$, while recognizing that the intermediate organolithium species might be in equilibrium with other tautomeric forms or with species having different levels of lithiation.

Table 1. Lithiation of 6 followed by reaction with benzophenone according to Scheme 4 under various reaction conditions

\begin{tabular}{cccc}
\hline Entry & $\mathrm{RLi}(\mathrm{mol}$ equiv) & $\mathrm{T}\left({ }^{\circ} \mathrm{C}\right)$ & ${\text { Yield }(\%)^{a}}^{a}$ \\
\hline 1 & $n-\operatorname{BuLi}(2.5)$ & -78 & $7^{b}$ \\
2 & $t$-BuLi (2.5) & -78 & $-{ }^{b}$ \\
3 & $n-\operatorname{BuLi}(2.5)$ & -20 & $17^{b}$ \\
4 & $t$-BuLi (2.5) & -20 & $31^{b}$ \\
5 & $n-\operatorname{BuLi}(2.5)$ & 0 & $--^{b, c}$ \\
6 & $t$-BuLi (2.5) & 0 & $-^{b, c}$ \\
7 & $t$-BuLi (3.3) & -40 to -30 & $51^{b}$ \\
8 & $t$-BuLi (3.3)/TMEDA (1.1) & -40 to -30 & 93 \\
\hline
\end{tabular}

${ }^{a}$ Yield by ${ }^{1} \mathrm{H}$ NMR. ${ }^{b}$ Starting material 6 was seen in the product mixture $\left({ }^{1} \mathrm{H}\right.$ NMR $) .{ }^{c}$ Sideproduct 10 was isolated, after purification by column chromatography (EtOAc), in 16 and 39\% yields, with $n$-BuLi and $t$-BuLi, respectively.

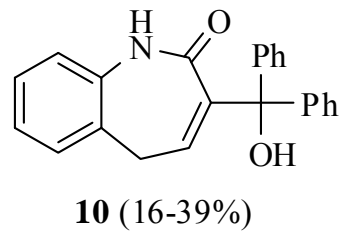

Figure 1. Structure of side-product (10). 
<smiles>CNC(=O)Nc1ccccc1C[Al]</smiles>

tautomer of 8

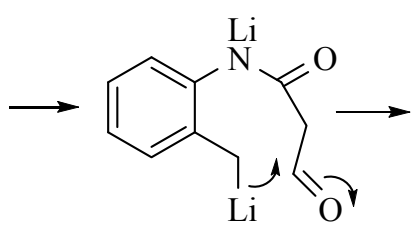<smiles></smiles>
$\mathrm{O}^{-} i^{+}$<smiles>O=C(NCCOC1=CC(O)Cc2ccccc2N1)c1ccccc1</smiles><smiles>O=C1Nc2ccccc2CC(O)C1C([O-])(c1ccccc1)c1ccccc1</smiles>
$\stackrel{\text { work up }}{\longrightarrow}$<smiles>O=C1Nc2ccccc2CC(O)C1C(O)(c1ccccc1)c1ccccc1</smiles>
$\stackrel{-\mathrm{H}_{2} \mathrm{O}}{\longrightarrow}$<smiles>O=C1Nc2ccccc2CC=C1C(O)(c1ccccc1)c1ccccc1</smiles>

10

Scheme 5. A possible mechanism for formation of $\mathbf{1 0 .}$

In order to avoid formation of the side product and to maximize the yield of $\mathbf{9}$ further reactions were conducted with $t$-BuLi at lower temperature. The results indicated that the highest yield of 9 was obtained by use of $t$-BuLi (3.3 equiv.) in the presence of tetramethylethylenediamine (TMEDA; 1.1 equiv.) at -40 to $-30{ }^{\circ} \mathrm{C}$ (Table 1; Entry 8), which gave 9 in $93 \%$ yield after crystallization, while use of $t$-BuLi without TMEDA under similar conditions produced 9 in 51\% yield along with unreacted 6 (Table 1; Entry 7).

Production of $\mathbf{9}$ in high yield implied that dilithium intermediate $\mathbf{8}$ had been formed efficiently. It was therefore interesting to see if reactions of $\mathbf{8}$ with other electrophiles would be useful, making the reaction general. Therefore, reactions of $\mathbf{8}$, prepared in-situ from compound $\mathbf{6}$, with other electrophiles (cyclohexanone, acetophenone, 2-butanone, benzaldehyde and iodomethane) were carried out. Each reaction was conducted under identical conditions and then quenched by the addition of aqueous $\mathrm{NH}_{4} \mathrm{Cl}$. Afterwards, the crude products were crystallized (Et ${ }_{2} \mathrm{O}$-hexane, 1:2 by volume) to give the corresponding substituted derivatives 11-15 (Scheme 6) in high yields (Table 2).
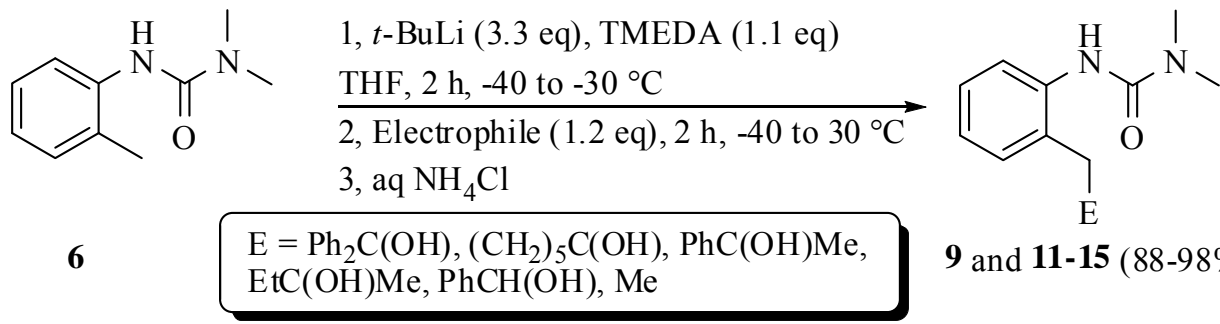

9 and 11-15 (88-98\%)

Scheme 6. Lateral lithiation and substitution of 6. 
Table 2. Synthesis of $N^{\prime}$-(2-(substituted methyl)phenyl)- $N, N$-dimethylureas 9 and 11-15 according to Scheme 6

\begin{tabular}{cccc}
\hline Product & Electrophile & $\mathrm{E}$ & Yield (\%) $^{a}$ \\
\hline $\mathbf{9}$ & $\mathrm{Ph}_{2} \mathrm{CO}$ & $\mathrm{Ph}_{2} \mathrm{C}(\mathrm{OH})$ & 93 \\
$\mathbf{1 1}$ & $\left(\mathrm{CH}_{2}\right)_{5} \mathrm{CO}$ & $\left(\mathrm{CH}_{2}\right)_{5} \mathrm{C}(\mathrm{OH})$ & 94 \\
$\mathbf{1 2}$ & $\mathrm{PhCOMe}$ & $\mathrm{PhC}(\mathrm{OH}) \mathrm{Me}$ & 91 \\
$\mathbf{1 3}$ & $\mathrm{EtCOMe}$ & $\mathrm{EtC}(\mathrm{OH}) \mathrm{Me}$ & 90 \\
$\mathbf{1 4}$ & $\mathrm{PhCHO}$ & $\mathrm{PhCH}(\mathrm{OH})$ & 98 \\
$\mathbf{1 5}$ & $\mathrm{MeI}$ & $\mathrm{Me}$ & 88 \\
\hline
\end{tabular}

${ }^{a}$ Yield of isolated product after crystallization ( $\mathrm{Et}_{2} \mathrm{O}-$ hexane, 1:2 by volume).

As can be seen from Table 2, the process is successful with various electrophiles. The ${ }^{1} \mathrm{H}$ NMR spectra of compounds 12-14 showed that the signals of the two protons of the $\mathrm{CH}_{2}$ group appeared separately, verifying that they are diastereotopic.

\section{Conclusions}

A simple, efficient and general procedure that allows lateral lithiation and substitution of $N^{\prime}-(2-$ methylphenyl)- $N, N$-dimethylurea has been demonstrated to provide high yields of various derivatives substituted on the 2-methyl group.

\section{Experimental Section}

General. Melting point determinations were performed by the open capillary method using a Gallenkamp melting point apparatus. ${ }^{1} \mathrm{H}$ spectra were recorded on a Bruker AV400 instrument operating at $400 \mathrm{MHz}$ and ${ }^{13} \mathrm{C}$ NMR spectra were recorded on a Bruker AV500 spectrometer operating at $125 \mathrm{MHz}$. Chemical shifts $\delta$ are reported in parts per million (ppm) relative to TMS and coupling constants $J$ are in $\mathrm{Hz} .{ }^{13} \mathrm{C}$ multiplicities were revealed by DEPT signals. Assignments of signals are based on integration values, coupling patterns and expected chemical shift values and have not been rigorously confirmed. Signals with similar characteristics might be interchanged. Low-resolution mass spectra were recorded on a Waters GCT Premier spectrometer and high-resolution mass spectra were recorded on a Waters LCT Premier XE instrument. IR spectra were recorded on a Perkin Elmer Spectrum One FT-IR spectrometer or a Perkin Elmer 1600 series FT-IR Spectrometer. Column chromatography was carried out using Fischer Scientific silica 60A (35-70 micron). Alkyllithiums were obtained from Aldrich 
Chemical Company and were estimated prior to use by the method of Watson and Eastham. ${ }^{25}$ Other chemicals were obtained from Aldrich Chemical Company and used without further purification.

$N^{\prime}$-(2-Methylphenyl)- $N, \boldsymbol{N}$-dimethylurea (6). A stirred mixture of 2-toluidine (5; $5.00 \mathrm{~g}, 46.7$ $\mathrm{mmol})$, dimethylcarbamoyl chloride $(6.04 \mathrm{~g}, 56.4 \mathrm{mmol})$ and triethylamine $(7.08 \mathrm{~g}, 70.0 \mathrm{mmol})$ in DCM $(70 \mathrm{~mL})$ was heated under reflux for $2 \mathrm{~h}$. The mixture was poured onto $\mathrm{H}_{2} \mathrm{O}(100 \mathrm{~mL})$ and the organic layer was separated, washed with $\mathrm{H}_{2} \mathrm{O}(2 \times 20 \mathrm{~mL})$, dried $\left(\mathrm{MgSO}_{4}\right)$ and evaporated under reduced pressure. The solid obtained was crystallized from a mixture of hexane/EtOAc/ $\mathrm{Et}_{2} \mathrm{O}$ (2/1/1 by volume) to give 6 (8.23 g, $\left.46.2 \mathrm{mmol}, 99 \%\right)$ as a white crystalline solid. Mp: $147-148{ }^{\circ} \mathrm{C}$ (lit. $\left.{ }^{23} 143-144{ }^{\circ} \mathrm{C}\right)$.

General procedure for lateral lithiation and substitution of $\boldsymbol{N}^{\prime}$-(2-methylphenyl)- $N, \boldsymbol{N}$ dimethylurea (6). A solution of $t-\mathrm{BuLi}$ in pentane $(1.95 \mathrm{~mL}, 1.90 \mathrm{M}, 3.70 \mathrm{mmol})$ was added to a stirred solution of $6(0.20 \mathrm{~g}, 1.12 \mathrm{mmol})$ at -40 to $-30{ }^{\circ} \mathrm{C}$ in anhydrous THF $(10 \mathrm{~mL})$ under a $\mathrm{N}_{2}$ atmosphere. TMEDA $(0.19 \mathrm{~mL}, 1.27 \mathrm{mmol})$ was added and the deep yellow solution was stirred at -40 to $-30{ }^{\circ} \mathrm{C}$ for $2 \mathrm{~h}$. The electrophile $(1.35 \mathrm{mmol})$, in anhydrous THF $(5 \mathrm{~mL})$ if solid, neat otherwise, was added. The reaction mixture was stirred for $2 \mathrm{~h}$ at -40 to $-30{ }^{\circ} \mathrm{C}$, and then allowed to warm to room temperature. The reaction mixture was quenched with a saturated aqueous solution of $\mathrm{NH}_{4} \mathrm{Cl}(10 \mathrm{~mL})$ and diluted with diethyl ether $(20 \mathrm{~mL})$. The organic layer was separated, washed with $\mathrm{H}_{2} \mathrm{O}(2 \times 20 \mathrm{~mL})$, dried $\left(\mathrm{MgSO}_{4}\right)$ and evaporated under reduced pressure. The crude product was purified by crystallization $\left(\mathrm{Et}_{2} \mathrm{O}\right.$-hexane, 1:2 by volume) to give the pure products. The yields obtained were in the range of 88-98\% (Table 2).

$N^{\prime}$-[2-(2-Hydroxy-2,2-diphenylethyl)phenyl]- $N, \boldsymbol{N}$-dimethylurea (9). Yield: $0.376 \mathrm{~g}$ (1.04 mmol, 93\%); white solid; Mp 218-219 ${ }^{\circ} \mathrm{C}$. IR (FT): $v_{\max } 3348,2937,1631,1519,1445 \mathrm{~cm}^{-1} .{ }^{1} \mathrm{H}$ NMR (400 MHz, $\mathrm{CDCl}_{3}$ ): $\delta=8.15$ (s, exch., $\left.1 \mathrm{H}, \mathrm{NH}\right), 7.67$ (dd, J 1.2, 8.0 Hz, $1 \mathrm{H}, \mathrm{H}-6$ ), 7.347.30 (m, $11 \mathrm{H}, 2 \mathrm{Ph}$ and $\mathrm{OH}$ ), 7.27 (app. dt, J 1.2, 8.0 Hz, $1 \mathrm{H}, \mathrm{H}-5$ ), 6.70 (app. dt, J 1.2, 8.0 Hz,

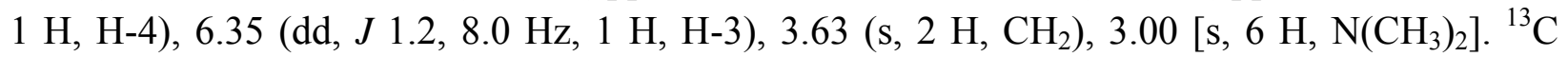
NMR (125 MHz, CDCl $\left.)_{3}\right): \delta=156.6(\mathrm{~s}, \mathrm{C}=\mathrm{O}), 146.0(\mathrm{~s}, \mathrm{C}-1$ of $2 \mathrm{Ph}), 142.7(\mathrm{~s}, \mathrm{C}-1), 138.9(\mathrm{~s}, \mathrm{C}-$ 2), 132.2 (d, C-4), 128.2 (d, C-3/C-5 of 2 Ph), 127.5 (d, C-4 of 2 Ph), 127.0 (d, C-5), 126.4 (d, C2/C-6 of $2 \mathrm{Ph}$ ), 124.3 (d, C-3), 122.9 (d, C-6), 81.2 (s, C-OH), 43.9 (t, $\left.\mathrm{CH}_{2}\right), 36.5$ [q, N( $\left.\left.\mathrm{CH}_{3}\right)_{2}\right]$. MS $\left(\mathrm{ES}^{+}\right): m / z(\%)=399\left([\mathrm{M}+\mathrm{K}]^{+}, 11\right), 383\left([\mathrm{M}+\mathrm{Na}]^{+}, 100\right), 361\left([\mathrm{M}+\mathrm{H}]^{+}, 31\right), 343(55)$, 315 (12). HRMS (ES $\left.{ }^{+}\right): m / z$ calcd for $\mathrm{C}_{23} \mathrm{H}_{25} \mathrm{~N}_{2} \mathrm{O}_{2}[\mathrm{M}+\mathrm{H}]^{+}: 361.1916$; found: 361.1927 .

3-(Hydroxydiphenylmethyl)-1,5-dihydro-2H-1-benzazepin-2-one (10). Yield: $0.061-0.149 \mathrm{~g}$ (0.179-0.44 mmol, 16-39\%); brownish oil. IR (FT): $v_{\max } 3352,2948,1635,1593,1522,1438$ $\mathrm{cm}^{-1} .{ }^{1} \mathrm{H}$ NMR $\left(400 \mathrm{MHz}, \mathrm{CDCl}_{3}\right.$ ): $\delta=7.55$ (d, J $\left.7.7 \mathrm{~Hz}, 1 \mathrm{H}, \mathrm{H}-9\right), 7.53$ (br, exch., $\left.1 \mathrm{H}, \mathrm{NH}\right)$, 7.39 (d, J 7.3 Hz, 4 H, H-2/H-6 of 2 Ph), 7.28 (app. t, J 7.3 Hz, 4 H, H-3/H-5 of 2 Ph), 7.20 (t, J $7.3 \mathrm{~Hz}, 2 \mathrm{H}, \mathrm{H}-4$ of $2 \mathrm{Ph}$ ), 7.16 (app. t, J $7.7 \mathrm{~Hz}, 1 \mathrm{H}, \mathrm{H}-8$ ), 6.98 (t, J 7.2 Hz, $1 \mathrm{H}, \mathrm{H}-4$ ), 6.96 (app. t, J 7.7 Hz, 1 H, H-7), 6.77 (d, J 7.7 Hz, 1 H, H-6), 3.58 (d, J 7.2 Hz, $2 \mathrm{H}, \mathrm{H}-5) .{ }^{13} \mathrm{C}$ NMR $\left(125 \mathrm{MHz}, \mathrm{CDCl}_{3}\right): \delta=162.5$ (s, C-2), 146.1 (s, C-1 of $\left.2 \mathrm{Ph}\right), 141.1$ (s, C-3), 137.1 (d, C-4), 129.9 (s, C-9a), 129.3 (d, C-8), 128.6 (d, C-3/C-5 of 2 Ph), 127.4 (d, C-4 of 2 Ph), 126.1 (d, C- 
2/C-6 of 2 Ph), 123.9 (d, C-6); 122.6 (s, C-5a), 122.2 (d, C-7), 110.1 (d, C-9), 78.2 (s, C-OH), $42.3(\mathrm{t}, \mathrm{C}-5)$. MS $\left.\left(\mathrm{ES}^{+}\right): \mathrm{m} / \mathrm{z}(\%)=382\left(\left[\mathrm{M}+\mathrm{CH}_{3} \mathrm{CN}\right]^{+}, 100\right), 380(\mathrm{M}+\mathrm{K}]^{+}, 47\right), 364([\mathrm{M}+$ $\left.\mathrm{Na}]^{+}, 27\right), 324$ (12), 107 (5). HRMS $\left(\mathrm{ES}^{+}\right): \mathrm{m} / \mathrm{z}$ calcd for $\mathrm{C}_{23} \mathrm{H}_{19} \mathrm{NO}_{2} \mathrm{Na}[\mathrm{M}+\mathrm{Na}]^{+}: 364.1313$; found: 364.1325 .

$N^{\prime}$-[2-[(1-Hydroxycyclohexyl)methyl]phenyl]- $N, N$-dimethylurea (11). Yield: $0.277 \mathrm{~g}$ (1.06 mmol, 94\%); white solid; Mp 159-160 ${ }^{\circ} \mathrm{C}$. IR (FT): $v_{\max }$ 3300, 2925, 1628, 1522, 1499, 1372 $\mathrm{cm}^{-1} .{ }^{1} \mathrm{H}$ NMR $\left(400 \mathrm{MHz}, \mathrm{CDCl}_{3}\right.$ ): $\delta=8.67$ (s, exch., $\left.1 \mathrm{H}, \mathrm{NH}\right), 8.03$ (dd, $J 1.2,7.6 \mathrm{~Hz}, 1 \mathrm{H}, \mathrm{H}-$ 6), 7.47 (app. dt, J 1.2, 7.6 Hz, 1 H, H-5), 7.30 (dd, J 1.2, 7.6 Hz, 1 H, H-3), 7.22 (app. dt, J 1.2, $7.6 \mathrm{~Hz}, 1 \mathrm{H}, \mathrm{H}-4), 3.27$ [s, $6 \mathrm{H}, \mathrm{N}\left(\mathrm{CH}_{3}\right)_{2}$ ], 3.02 (s, $\left.2 \mathrm{H}, \mathrm{CH}_{2}\right), 2.01$ (br, exch., $\left.1 \mathrm{H}, \mathrm{OH}\right), 1.87-$ $1.70\left(\mathrm{~m}, 10 \mathrm{H}\right.$, cyclohexyl). ${ }^{13} \mathrm{C} \mathrm{NMR}\left(125 \mathrm{MHz}, \mathrm{CDCl}_{3}\right): \delta=156.2(\mathrm{~s}, \mathrm{C}=\mathrm{O}), 137.0(\mathrm{~s}, \mathrm{C}-1)$, 133.1 (s, C-2), 131.9 (d, C-4), 127.2 (d, C-5), 124.0 (d, C-3), 122.7 (d, C-6), 74.3 (s, C-1 of cyclohexyl), 37.8 (t, C-2/C-6 of cyclohexyl), 36.5 [q, N( $\left.\left(\mathrm{CH}_{3}\right)_{2}\right], 35.4\left(\mathrm{t}, \mathrm{CH}_{2}\right), 25.6$ (t, C-4 of cyclohexyl), 22.35 (t, C-3/C-5 of cyclohexyl). MS (ES $): m / z(\%)=373$ (24), 339 (100), 276 $\left(\mathrm{M}^{-}, 21\right), 275\left([\mathrm{M}-\mathrm{H}]^{-}, 81\right), 177$ (18). HRMS $\left(\mathrm{ES}^{-}\right): \mathrm{m} / \mathrm{z}$ calcd for $\mathrm{C}_{16} \mathrm{H}_{23} \mathrm{~N}_{2} \mathrm{O}_{2}[\mathrm{M}-\mathrm{H}]^{-}$: 275.1760; found: 275.1765 .

$N^{\prime}$-[2-(2-Hydroxy-2-phenylpropyl)phenyl]- $N, N$-dimethylurea (12). Yield: 0.305 g (1.02 mmol, 91\%); white solid; Mp 158-160 ${ }^{\circ} \mathrm{C}$. IR (FT): $v_{\max }$ 3352, 2948, 1635, 1593, 1522, 1438 $\mathrm{cm}^{-1} .{ }^{1} \mathrm{H}$ NMR (400 MHz, $\mathrm{CDCl}_{3}$ ): $\delta=8.67$ (s, exch., $1 \mathrm{H}, \mathrm{NH}$ ), 8.06 (br d, J 7.6 Hz, $1 \mathrm{H}, \mathrm{H}-6$ ), 7.76 (d, J 7.5 Hz, 2 H, H-2/H-6 of Ph), 7.66 (app. t, J 7.5 Hz, 2 H, H-3/H-5 of Ph), 7.60 (t, J 7.5 $\mathrm{Hz}, 1 \mathrm{H}, \mathrm{H}-4$ of Ph), 7.56 (s, exch., $1 \mathrm{H}, \mathrm{OH}$ ), 7.52 (app. Dt, J 1.2, $7.6 \mathrm{~Hz}, 1 \mathrm{H}, \mathrm{H}-5$ ), 7.22 (app. dt, $J 1.2,7.6 \mathrm{~Hz}, 1 \mathrm{H}, \mathrm{H}-4), 7.16$ (dd, J 1.2, 7.6 Hz, $1 \mathrm{H}, \mathrm{H}-3), 3.38$ (d, J 14.2 Hz, $1 \mathrm{H}, \mathrm{CH}_{a} \mathrm{H}_{\mathrm{b}}$ ), $3.36\left[\mathrm{~s}, 6 \mathrm{H}, \mathrm{N}\left(\mathrm{CH}_{3}\right)_{2}\right], 3.21\left(\mathrm{~d}, J 14.2 \mathrm{~Hz}, 1 \mathrm{H}, \mathrm{CH}_{\mathrm{a}} \mathrm{H}_{b}\right), 1.93\left(\mathrm{~s}, 3 \mathrm{H}, \mathrm{CH}_{3}\right) .{ }^{13} \mathrm{C} \mathrm{NMR}(125 \mathrm{MHz}$, $\left.\mathrm{CDCl}_{3}\right): \delta=156.5(\mathrm{~s}, \mathrm{C}=\mathrm{O}), 148.3(\mathrm{~s}, \mathrm{C}-1$ of $\mathrm{Ph}), 136.1(\mathrm{~s}, \mathrm{C}-1), 134.5$ (s, C-2), $132.2(\mathrm{~d}, \mathrm{C}-4)$, 128.4 (d, C-3/C-5 of Ph), 127.3 (d, C-5), 127.1 (d, C-4 of Ph), 124.5 (d, C-2/C-6 of Ph), 124.2 (d, C-3), 122.9 (d, C-6), 77.5 (s, C-OH), 46.7 (t, $\left.\mathrm{CH}_{2}\right), 36.5$ [q, N( $\left.\left.\mathrm{CH}_{3}\right)_{2}\right], 28.9$ (q, $\left.\mathrm{CH}_{3}\right)$. MS (EI $\left.{ }^{+}\right)$: $\mathrm{m} / \mathrm{z}(\%)=280\left(\left[\mathrm{M}-\mathrm{H}_{2} \mathrm{O}\right]^{+}, 20\right), 265$ (13), 235 (17), 208 (24), 194 (33), 178 (13), 165 (11), 133 (12), 103 (18), 77 (16), 72 (100). HRMS $\left(\mathrm{EI}^{+}\right): \mathrm{m} / \mathrm{z}$ calcd for $\mathrm{C}_{18} \mathrm{H}_{20} \mathrm{~N}_{2} \mathrm{O}\left[\mathrm{M}-\mathrm{H}_{2} \mathrm{O}\right]^{+}: 280.1576$; found: 280.1577 .

$N^{\prime}$-[2-(2-Hydroxy-2-methylbutyl)phenyl]- $N, \boldsymbol{N}$-dimethylurea (13). Yield: $0.238 \mathrm{~g}$ (1.01 mmol, 90\%); white solid; Mp 111-112 ${ }^{\circ} \mathrm{C}$. IR (FT): $v_{\max } 3320,2950,1635,1532,1516,1373 \mathrm{~cm}^{-1} .{ }^{1} \mathrm{H}$ NMR (400 MHz, $\mathrm{CDCl}_{3}$ ): $\delta=7.64$ (s, exch., $\left.1 \mathrm{H}, \mathrm{NH}\right), 8.00$ (dd, J 1.2, $\left.7.6 \mathrm{~Hz}, 1 \mathrm{H}, \mathrm{H}-6\right), 7.45$ (app. dt, J 1.2, 7.6 Hz, 1 H, H-5), 7.25 (dd, J 1.2, 7.6 Hz, 1 H, H-3), 7.21 (app. dt, J 1.2, 7.6 Hz, 1 $\mathrm{H}, \mathrm{H}-4), 3.23$ [s, $\left.6 \mathrm{H}, \mathrm{N}\left(\mathrm{CH}_{3}\right)_{2}\right], 3.11$ (d, J $\left.14.1 \mathrm{~Hz}, 1 \mathrm{H}, \mathrm{PhCH}_{a} \mathrm{H}_{\mathrm{b}}\right), 2.85$ (d, J $14.2 \mathrm{~Hz}, 1 \mathrm{H}$, $\left.\mathrm{PhCH}_{\mathrm{a}} \mathrm{H}_{b}\right), 1.83\left(\mathrm{~m}, 3 \mathrm{H}, \mathrm{CH}_{3} \mathrm{CH}_{2}\right.$ and $\left.\mathrm{OH}\right), 1.42\left(\mathrm{~s}, 3 \mathrm{H}, \mathrm{CH}_{3}\right), 1.22$ (app. t, J $7.5 \mathrm{~Hz}, 3 \mathrm{H}$, $\left.\mathrm{CH}_{3} \mathrm{CH}_{2}\right) .{ }^{13} \mathrm{C}$ NMR $\left(125 \mathrm{MHz}, \mathrm{CDCl}_{3}\right): \delta=156.5(\mathrm{~s}, \mathrm{C}=\mathrm{O}), 138.9(\mathrm{~s}, \mathrm{C}-1), 132.1(\mathrm{~d}, \mathrm{C}-4)$, 128.6 (s, C-2), 127.2 (d, C-5), 124.1 (d, C-3), 122.8 (d, C-6), 75.9 (s, C-OH), 43.1 (t, $\mathrm{PhCH}_{2}$ ), 36.4 [q, N( $\left.\mathrm{CH}_{3}\right)_{2}$ ], $35.4\left(\mathrm{t}, \mathrm{CH}_{3} \mathrm{CH}_{2}\right), 26.6$ (q, $\left.\mathrm{CH}_{3}-\mathrm{C}\right), 8.4\left(\mathrm{q}, \mathrm{CH}_{3} \mathrm{CH}_{2}\right) . \mathrm{MS}\left(\mathrm{ES}^{-}\right): \mathrm{m} / \mathrm{z}(\%)=$ $250\left(\mathrm{M}^{-}, 15\right), 249$ ([M - H] $\left.]^{-}, 78\right), 177$ (100), 132 (15), 106 (10). HRMS (ES $\left.{ }^{-}\right): m / z$ calcd for $\mathrm{C}_{14} \mathrm{H}_{21} \mathrm{~N}_{2} \mathrm{O}_{2}[\mathrm{M}-\mathrm{H}]^{-}: 249.1603$; found: 249.1595 . 
$N^{\prime}$-[2-(2-Hydroxy-2-phenylethyl)phenyl]- $N, N$-dimethylurea (14). Yield: $0.313 \mathrm{~g}$ (1.10 mmol, 98\%); white solid; Mp 161-163 ${ }^{\circ} \mathrm{C}$. IR (FT): $v_{\max } 3245,2950,1629,1540,1378 \mathrm{~cm}^{-1} .{ }^{1} \mathrm{H}$ NMR $\left(400 \mathrm{MHz}, \mathrm{CDCl}_{3}\right): \delta=8.37$ (s, exch., $\left.1 \mathrm{H}, \mathrm{NH}\right), 7.99$ (d, J 8.1 Hz, $\left.1 \mathrm{H}, \mathrm{H}-6\right), 7.64-7.48$ (m, 7 H, Ph, H-3 and OH), 7.33-7.25 (m, 2 H, H-4 and H-5), 5.24 (dd, J 2.7, 9.4 Hz, $1 \mathrm{H}, \mathrm{CH}), 3.32$ [s, 6 $\left.\mathrm{H}, \mathrm{N}\left(\mathrm{CH}_{3}\right)_{2}\right], 3.20\left(\mathrm{dd}, J 9.4,14.4 \mathrm{~Hz}, 1 \mathrm{H}, \mathrm{CH}_{a} \mathrm{H}_{\mathrm{b}}\right), 3.16\left(\mathrm{dd}, J 2.7,14.4 \mathrm{~Hz}, 1 \mathrm{H}, \mathrm{CH}_{\mathrm{a}} \mathrm{H}_{b}\right) .{ }^{13} \mathrm{C}$ NMR (125 MHz, $\left.\mathrm{CDCl}_{3}\right): \delta=156.5(\mathrm{~s}, \mathrm{C}=\mathrm{O}), 142.5$ (s, C-1 of Ph), 138.5 (s, C-1), 133.5 (s, C2), 130.7 (d, C-4), 128.7 (d, C-3/C-5 of Ph), 128.0 (d, C-5), 127.3 (d, C-4 of Ph), 125.5 (d, C2/C-6 of Ph), 124.4 (d, C-3), 123.7 (d, C-6), 77.5 (d, CH), 42.0 (t, $\left.\mathrm{CH}_{2}\right), 36.5$ [q, N( $\left.\mathrm{CH}_{3}\right)_{2}$ ]. MS $\left(\mathrm{EI}^{+}\right): m / z(\%)=266\left(\left[\mathrm{M}-\mathrm{H}_{2} \mathrm{O}\right]^{+}, 28\right), 221$ (14), 194 (55), 165 (18), 133 (15), 118 (25), 107 (26), 77 (46), 72 (100). HRMS $\left(\mathrm{EI}^{+}\right)$: $\mathrm{m} / \mathrm{z}$ calcd for $\mathrm{C}_{17} \mathrm{H}_{18} \mathrm{~N}_{2} \mathrm{O}\left[\mathrm{M}-\mathrm{H}_{2} \mathrm{O}\right]^{+}:$266.1419; found: 266.1418 .

$N^{\prime}$-(2-Ethylphenyl)- $\boldsymbol{N}, \boldsymbol{N}$-dimethylurea (15). Yield: $0.190 \mathrm{~g}$ (0.99 mmol, 88\%); white solid; Mp 149-151 ${ }^{\circ} \mathrm{C}$. IR (FT): $v_{\max } 3270,2964,1636,1520,1448 \mathrm{~cm}^{-1} .{ }^{1} \mathrm{H}$ NMR $\left(400 \mathrm{MHz}, \mathrm{CDCl}_{3}\right): \delta=$ 7.66 (dd, J 1.2, 7.6 Hz, 1 H, H-6), 7.14-7.10 (m, 2 H, H-3 and H-5), 6.98 (app. dt, J 1.2, 7.6 Hz, $1 \mathrm{H}, \mathrm{H}-4), 6.11$ (s, exch., $1 \mathrm{H}, \mathrm{NH}), 2.98$ [s, $\left.6 \mathrm{H}, \mathrm{N}\left(\mathrm{CH}_{3}\right)_{2}\right], 2.54$ (q, J 7.6 Hz, $\left.2 \mathrm{H}, \mathrm{CH}_{2}\right), 1.18$ (t, $\left.J 7 \mathrm{~Hz}, 3 \mathrm{H}, \mathrm{CH}_{3}\right) .{ }^{13} \mathrm{C} \mathrm{NMR}\left(125 \mathrm{MHz}, \mathrm{CDCl}_{3}\right): \delta=156.1(\mathrm{~s}, \mathrm{C}=\mathrm{O}), 136.5(\mathrm{~s}, \mathrm{C}-1), 134.0(\mathrm{~s}, \mathrm{C}-$ 2), 128.3 (d, C-4), 126.7 (d, C-5), 124.1 (d, C-3), 123.0 (d, C-6), 36.5 [q, N(CH $)_{2}$ ], 24.4 (t, $\left.\mathrm{CH}_{2}\right), 13.8$ (q, $\left.\mathrm{CH}_{3}\right) . \mathrm{MS}\left(\mathrm{EI}^{+}\right): m / z(\%)=192\left(\mathrm{M}^{+}, 21\right), 147$ (32), 132 (25), 120 (24), 104 (7), 91 (8), 84 (14), 77 (13), 72 (100). HRMS (EI $\left.{ }^{+}\right): m / z$ calcd for $\mathrm{C}_{11} \mathrm{H}_{16} \mathrm{~N}_{2} \mathrm{O}$ [M] $]^{+}:$192.1263; found: 192.1259 .

\section{Acknowledgements}

This project was supported by the Deanship of Scientific Research at Salman bin Abdulaziz University under the research project 2013/01/8 and Al-Qasim Green University.

\section{References}

1. Clark, R. D.; Jahangir, A. In Organic Reactions, Paquette, L. A. Ed.; Wiley: New York, 1995, Vol. 47, p 1.

2. Clayden, J. Organolithiums: Selectivity for Synthesis, Pergamon: Oxford, 2002.

3. Katritzky, A. R.; Akutagawa, K. J. Am. Chem. Soc. 1986, 108, 6808-6809. http://dx.doi.org/10.1021/ja00281a061

4. Katritzky, A. R.; Black, M.; Fan, W.-Q. J. Org. Chem. 1991, 56, 5045-5048. http://dx.doi.org/10.1021/jo00017a012

5. Clark, R. D.; Muchowski, J. M.; Fisher, L. E.; Flippin, L. A.; Repke, D. B.; Souchet, M. Synthesis 1991, 871-878.

http://dx.doi.org/10.1055/s-1991-26597 
6. Sibi, M. P.; Snieckus, V. J. Org. Chem. 1983, 48, 1935-1937.

http://dx.doi.org/10.1021/jo00159a040

7. Clayden, J.; Dufour, J. Tetrahedron Lett. 2006, 47, 6945-6946. http://dx.doi.org/10.1016/j.tetlet.2006.07.134

8. Uchida, K.; Fukuda, T.; Iwao, M. Tetrahedron 2007, 63, 7178-7186. http://dx.doi.org/10.1016/j.tet.2007.04.092

9. Clayden, J.; Dufour, J.; Grainger, D. M.; Helliwell, M. J. Am. Chem. Soc. 2007, 129, 74887489.

http://dx.doi.org/10.1021/ja071523a

10. Wilkinson, J. A.; Raiber, E.-A.; Ducki, S. Tetrahedron Lett. 2007, 48, 6434-6436. http://dx.doi.org/10.1016/j.tetlet.2007.07.070

11. Burgos, P. O.; Fernández, I.; Iglesias, M. J.; García-Granda, S.; Ortiz, F. L. Org. Lett. 2008, 10, 537-540.

http://dx.doi.org/10.1021/o17028096

12. Marcos, I. S.; Beneitez, A.; Moro, R. F.; Basabe, P.; Díez, D.; Urones, J. G. Tetrahedron 2010, 66, 7773-7780. http://dx.doi.org/10.1016/j.tet.2010.07.066

13. Kacem, Y.; Ben Hassine, B. Tetrahedron Lett. 2012, 53, 5608-5610. http://dx.doi.org/10.1016/j.tetlet.2012.08.008

14. Jana, A. K.; Pahari, P.; Mal, D. Synlett 2012, 23, 1769-1774. http://dx.doi.org/10.1055/s-0031-1290380

15. Slabu, I.; Rossington, S. B.; Killoran, P. M.; Hirst, N.; Wilkinson, J. A. Tetrahedron Lett. 2013, 54, 1489-1490.

http://dx.doi.org/10.1016/j.tetlet.2013.01.040

16. Giovine, A.; Musio, B.; Degennaro, L.; Falcicchio, A.; Nagaki, A.; Yoshida, J.-I.; Luisi, R. Chem. Eur. J. 2013, 19, 1872-1876. http://dx.doi.org/10.1002/chem.201203533

17. Smith, K.; El-Hiti, G. A.; Shukla, A. P. J. Chem. Soc., Perkin Trans. 1 1999, 2305-2313. http://dx.doi.org/10.1039/A903464A

18. Smith, K.; El-Hiti, G. A.; Abdo, M. A.; Abdel-Megeed, M. F. J. Chem. Soc., Perkin Trans. 1 1995, 1029-1033. http://dx.doi.org/10.1039/P19950001029

19. Smith, K.; El-Hiti, G. A.; Abdel-Megeed, M. F.; Abdo, M. A. J. Org. Chem. 1996, 61, 656661. http://dx.doi.org/10.1021/j09509891

20. Smith, K.; El-Hiti, G. A.; Abdel-Megeed, M. F. Synthesis 2004, 2121-2130. http://dx.doi.org/10.1055/s-2004-829169

21. Smith, K.; El-Hiti, G. A.; Hegazy, A. S. Synthesis 2010, 1371-1380. http://dx.doi.org/10.1055/s-0029-1219277 
22. Smith, K.; El-Hiti, G. A.; Alshammari, M. B. J. Org. Chem. 2012, 77, 11210-11215. http://dx.doi.org/10.1021/jo3023445

23. Houlden, C. E.; Bailey, C. D.; Ford, J. G.; Gagné, M. R.; Lloyd-Jones, G. C.; BookerMilburn, K. I. J. Am. Chem. Soc. 2008, 130, 10066-10067.

http://dx.doi.org/10.1021/ja803397y

24. Clayden, J.; Yasin, S. A. New J. Chem. 2002, 26, 191-192. http://dx.doi.org/10.1039/B109604D

25. Watson, S. C.; Eastham, J. F. J. Organomet. Chem. 1967, 9, 165-168. http://dx.doi.org/10.1016/S0022-328X(00)92418-5 